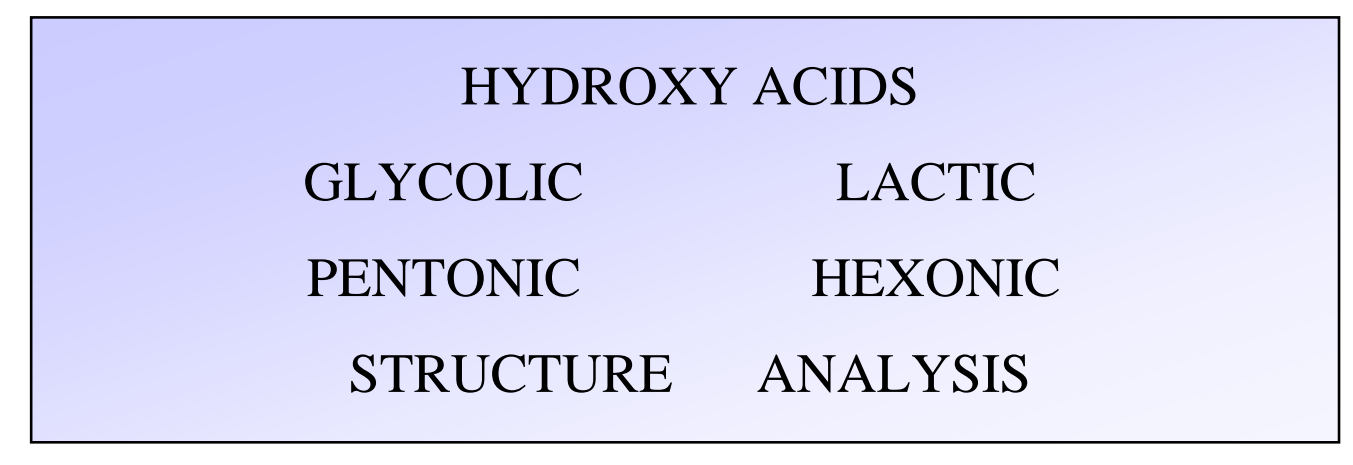

Article included in $\underline{\mathrm{PhD} \text { thesis }}$

Tetrahedron 26 (1970) 3413-3428

Link to publisher: doi:10.1016/S0040-4020(01)92918-7

\title{
Mass spectrometry of aldonic and deoxyaldonic acids as trimethylsilyl derivatives
}

\author{
Göran Petersson \\ Department of Engineering Chemistry, Chalmers University of Technology, \\ Göteborg, Sweden
}

Related articles:

Mass spectrometric identification of aldonolactones

Mass spectrometry of hydroxy dicarboxylic acids 


\begin{abstract}
Open-chain trimethylsilyl derivatives of aldonic and deoxyaldonic acids, which can be prepared from salts of these hydroxy acids, are well suited for structure determination by mass spectrometry. The study is focused on the aldonic and unbranched monodeoxyaldonic acids, but several dideoxyaldonic and branched deoxyaldonic acids are included. Spectra of the derivatives of glycolic, lactic and hydracrylic acids are discussed.

The structural isomers exhibit interpretable differences, whereas the spectra of the diastereomers are similar. The molecular weight and the number of $\mathrm{OH}$ groups can be deduced from the spectra, as well as the position of the "deoxy group” in unbranched deoxyaldonic acids. The most prominent fragmentations are $\alpha$-cleavages of the carbon chain, frequently followed by rearrangement loss of trimethylsilanol. A structurally significant McLafferty-type rearrangement of a trimethylsilyl group is described. Several types of ions decompose by expulsion of small molecules such as $\mathrm{CO}, \mathrm{CH}_{2} \mathrm{O}$ and $\mathrm{CO}_{2}$.
\end{abstract}

\title{
Sammanfattning
}

Trimetylsilylderivat av aldonsyror och deoxialdonsyror kan framställas från salter av syrorna och passar väl för strukturbestämning med masspektrometri. Studien fokuserar på aldonsyror och oförgrenade monodeoxialdonsyror men flera dideoxialdonsyror och grenade deoxialdonsyror har inkluderats. Spektra för derivaten av glykolsyra, mjölksyra och hydrakrylsyra diskuteras.

Strukturisomerer ger tolkningsbara skillnader, medan spektra av diastereomerer är likartade. Molekylvikten och antalet $\mathrm{OH}$-grupper kan härledas från spektra liksom positionen av deoxigrupperingen i acykliska deoxialdonsyror. De mest framträdande fragmenteringarna är $\alpha$-klyvningar av kolkedjan som ofta följs av avspaltning av trimetylsilanol. En specifik omlagring av McLafferty-typ för en trimetylsilylgrupp beskrivs. Flera slag av joner sönderfaller genom avspaltning av små molekyler som $\mathrm{CO}, \mathrm{CH}_{2} \mathrm{O}$ och $\mathrm{CO}_{2}$. 


\title{
Mass spectrometry of aldonic and deoxyaldonic acids as trimethylsilyl derivatives
}

\author{
Göran Petersson \\ Department of Engineering Chemistry, \\ Chalmers University of Technology, \\ Göteborg, Sweden
}

TRIMETHYLSILYL (TMS) derivatives of aldonolactones have proven very useful for mass spectrometric identification of aldonic acids ${ }^{1}$ though their fragmentation has not been explained in detail. A preliminary report of the mass spectra of fully trimethylsilylated TMS esters of pentonic and hexonic acids ${ }^{2}$ demonstrated a more easily understood relationship between structures and spectra for these derivatives. The most important primary fragmentations were shown to be carbon chain cleavages, as could be expected from the well-known preference for $\alpha$-cleavages in the fragmentation of ethers.

The favoured formation of ions from $\alpha$-cleavages suggested that these derivatives might be suitable for structure determinations of deoxyaldonic acids as well, and a systematic study of aldonic and deoxyaldonic acids was begun. A comparison with the spectra of TMS derivatives of alditols ${ }^{3}$ aids in the interpretation. Some fragmentation analogies with trimethylsilylated long-chain hydroxy acid methyl esters are also found. The formation of abundant $\alpha$-cleavage ions has been used to determine the position of hydroxyl groups for acids of this type. ${ }^{4-7}$

The open-chain TMS derivatives were prepared rapidly and conveniently from salts of the acids. Derivatization of carboxyl groups by this method has been reported for other types of carboxylic acids.8,9. One single derivative form is obtained which is often a great advantage in separations by gas chromatography of mixtures containing lactone-forming acids.

The present investigation was carried out with emphasis on acids formed from cellulosic materials in various technical processes and on hydrolysis. It includes aldonic acids, 2-deoxy-and 3-deoxyaldonic acids, a few $\omega$-deoxy-and dideoxyaldonic 


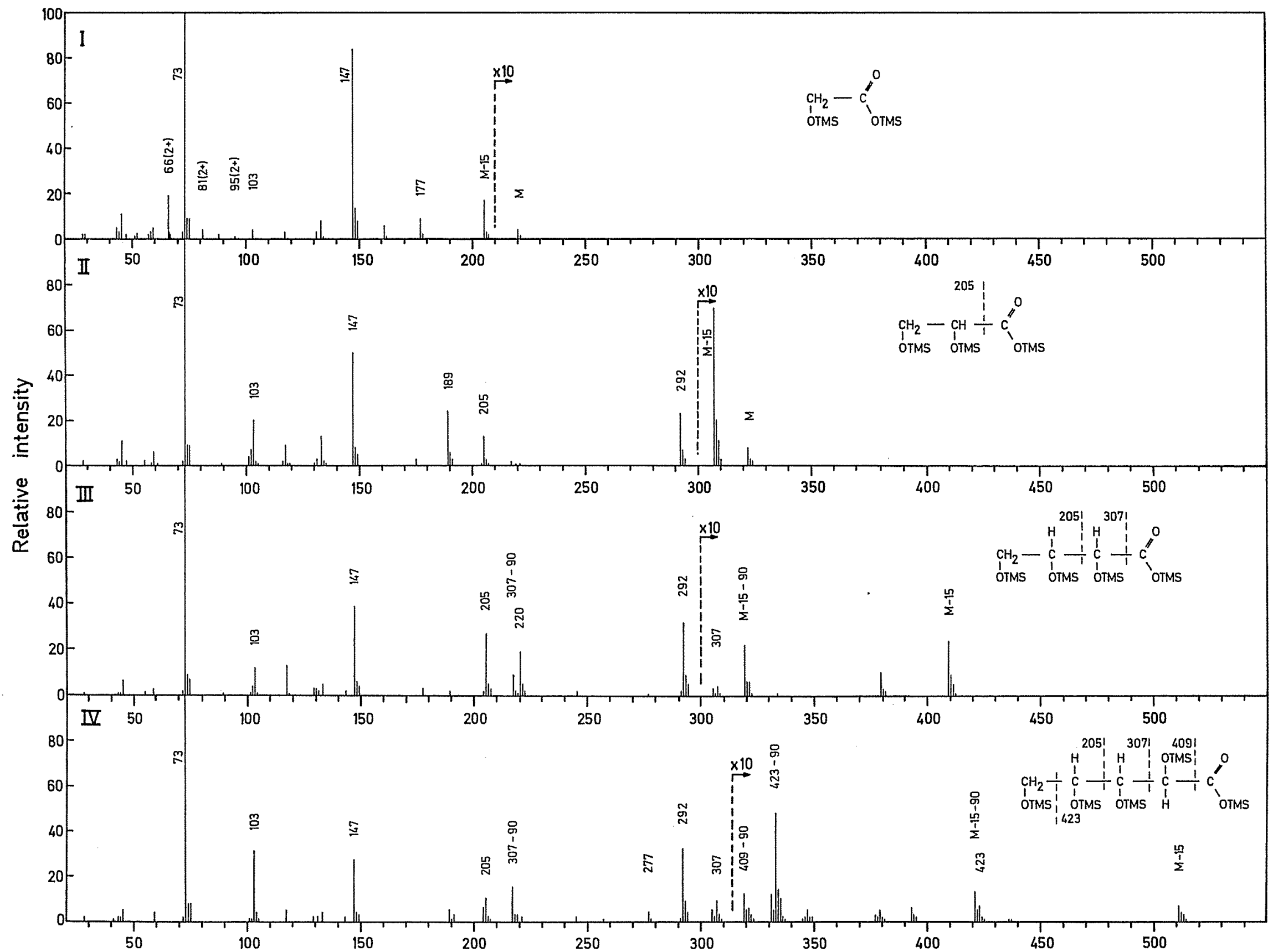

FIG 1. Mass spectra at $70 \mathrm{eV}$ of the trimethylsilyl (TMS) derivatives of glycolic (I), glyceric (II), erythronic (III) and arabinonic (IV) acids 
acids and the most common branched-chain saccharinic acids. The fragmentation is discussed and the most characteristic mass spectral features resulting from the different structures are pointed out.

Since enantiomers give identical mass spectra the enantiomeric configurations have been omitted and the $\mathrm{D}$-forms have been chosen arbitrarily for the structure formulas of diastereomeric acids.

\section{Glycolic, hydracrylic and lactic acids}

The spectrum of the TMS derivative of glycolic acid is given in Fig 1, of hydracrylic (2-deoxyglyceric) acid in Fig 4 and of lactic (3-deoxyglyceric) acid in Fig 5. Because of several obvious fragmentation analogies the spectra of these acids are discussed here as a group rather than in connection with the spectra of their higher analogs.
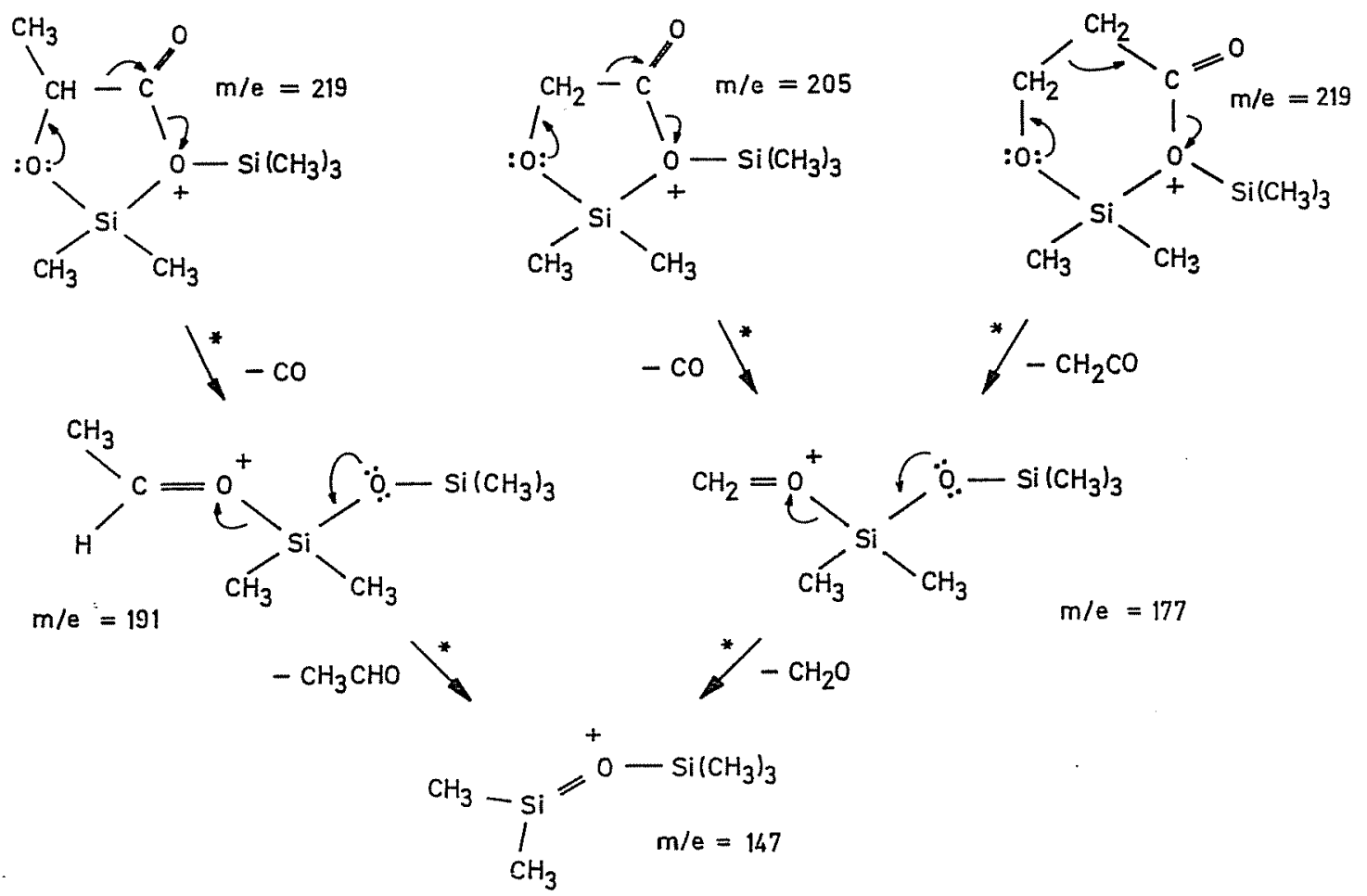

FIG 2. Decomposition of M-15 ions for lactic, glycolic and hydracrylic acids

Small peaks from the molecular ions are obtained for glycolic and hydracrylic acids, but the most significant peak that can be directly related to the molecular weight is found at M-15. It is due to the well-known favoured loss in TMS derivatives of a methyl group linked to silicon.

Of the expected peaks from $\mathrm{C}-\mathrm{C} \alpha$-cleavages the one at $m / e 117$ for lactic acid has a high intensity, whereas those at $m / e 103$ for glycolic and hydracrylic acids are of low intensity. For comparison, the spectra of the TMS derivatives of 2-hydroxybutyric and 2-hydroxyisovaleric acids were studied. The corresponding peaks at $m / e 131$ and 145 were found to be the most intense in these spectra. Metastable peaks indicated decomposition of the ions with mass 117,131 and 145 by loss of aldehydes as an important fragmentation path in the formation of the abundant trimethylsilyl ion with mass 73. The formation of this ion from $\mathrm{m} / e 103$ and $\mathrm{m} / \mathrm{e} 117$ ions for the derivatives of 1- and 2-pentanol has been demonstrated by Djerassi et al. ${ }^{10}$ and, in addition to the differences in structure, different decomposition rates may be responsible for the low 


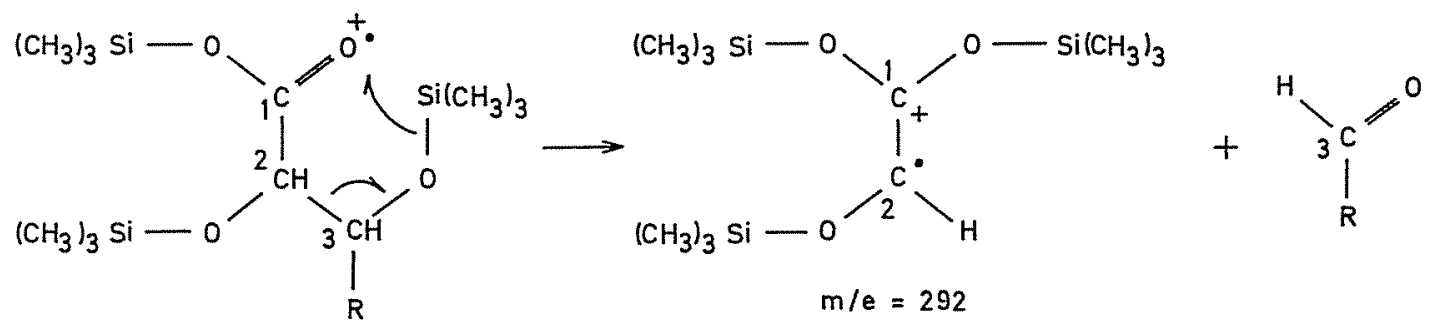

FIG 3. Formation of the $m / e 292$ ion by a McLafferty rearrangement of a trimethylsilyl group

intensity of the peaks at $m / e 103$ compared to the peak at $m / e 117$. A similar intensity ratio for these peaks was obtained in a study of TMS derivatives of a number of 1 - and 2-alkanols. ${ }^{11}$

A predominant fragmentation occurs, as illustrated in Fig 2, through rearrangement decomposition of the M-15 ions which can be depicted as cyclic oxonium ions. ${ }^{12}$ The abundant $m / e 147$ ion is formed by sequential loss of $\mathrm{CO}$ and $\mathrm{CH}_{2} \mathrm{O}$ for glycolic acid, $\mathrm{CO}$ and $\mathrm{CH}_{3} \mathrm{CHO}$ for lactic acid and $\mathrm{CH}_{2} \mathrm{CO}$ and $\mathrm{CH}_{2} \mathrm{O}$ for hydracrylic acid. As indicated by the asterisks, all the decomposition steps are supported by distinct metastable peaks. The formation of ions with charge stabilization from oxygen, analogous to that in ions from $\alpha$-cleavage, offers a plausible explanation for the loss of ketene from the 3-hydroxy acid as opposed to the loss of carbon monoxide from the 2hydroxy acids in the first fragmentation step. As could be expected, 2-hydroxybutyric and 2-hydroxyisovaleric acids exhibit peaks at M-43 with metastable peaks supporting the loss of carbon monoxide. Analogous fragmentations to those in Fig 2 for glycolic and lactic acids have been reported for the methyl esters of trimethylsilylated 2hydroxy acids ${ }^{5}$ and for various thioglycolic acid derivatives. ${ }^{13}$ Instead of occurring through decomposition of cyclic M-15 ions, the first fragmentation step may occur through direct migration of the ester trimethylsiloxyl group to a positive charge site at the other silicon atom. Loss of ketene comparable to that for hydracrylic acid has been pictured as similar migrations to carbonium ion centres for dimethyl esters ${ }^{14}$ and for $\beta$-(alkylthio)propionic acids. ${ }^{15}$ The formation of the $m / e 177$ ion by rearrangement and its decomposition to the $m / e 147$ ion has been reported for TMS derivatives of glycols. ${ }^{12}$ The formation of $m / e 147$ in the second fragmentation step occurs through heterolytic aldehyde loss and is comparable with the formation of the $m / e 73$ ion from the $\alpha$-cleavage ions. In addition to the two-step formation of the $m / e 147$ ion a metastable peak for glycolic acid indicates its formation directly from the M-15 ions. It should also be noted that fragmentation according to Fig 2 implies formation of the M-15 ions by loss of a methyl group from the ether TMS group. The alternative loss from the ester TMS group would give less favourable structures for the fragmentation products.

The presence of a number of rather intense peaks from doubly charged ions was indicated by half-mass isotope peaks and by unusual mass numbers. These ions probably originate from the loss of one methyl group at each of the two silicon atoms with the generation of two positively charged sites. Such ions have been observed previously in spectra of TMS esters. ${ }^{16}$ The masses of most of the ions are related to those of the ions arising from the decomposition of $\mathrm{M}-15$ ions, though cyclic intermediates seem improbable because of charge repulsion. Glycolic acid exhibits peaks at $m / e 95[\mathrm{M}-2 \times 15]^{2+}$ and $81[\mathrm{M}-2 \times 15-28]^{2+}$, lactic acid at $m / e 102[\mathrm{M}-2 \times 15]^{2+}$ and 
$88[-2 \times 15-28]^{2+}$ and hydracrylicacid at $m / e 102[\mathrm{M}-2 \times 15]^{2+}, 81[\mathrm{M}-2 \times 15-42]^{2+}$ and $87[\mathrm{M}-2 \times 15-30]^{2+}$. The most intense peak of this kind for the three acids if found at $m / e \quad 66$ and is obtained for the 2-hydroxybutyric and 2-hydroxyisovaleric acids as well. It should be the ion corresponding to the $m / e 147$ ion minus a Me group. A metasable peak for glycolic acid at $m / e 81$ ion by loss of formaldehyde. No peaks from doubly charged ions were observed in the spectra of the other acids investigated. This difference can be associated with the presence of energetically more favourable fragmentation pathways for these acids.

The only abundant odd-electron ion in the three spectra gives rise to a peak at $m / e$ 190 for lactic acid. The formation of this ion by the loss of acetaldehyde is suggested by the presence of the same peak in the spectra of the 2-hydroxybutyric and 2hydroxyisovaleric acids. The fragmentations discussed were found to explain all intense peaks in the spectra of these two acids as well.

\section{Aldonic acids}

Spectra of the TMS derivatives of glyceric acid, of all the diastereomeric tetronic, pentonic and hexonic acids and of D-glycero-D-gulo-heptonic acid were recorded. Illustrative spectra for glyceric, erythronic and arabinonic acids are given in Fig 1, below that of glycolic acid. The spectrum of the mannonic acid derivative reproduced in an earlier report ${ }^{2}$ is representative for hexonic acids. The formation of abundant ions originating from cleavages in the carbon chain and the presence of a significant peak at M-15 were pointed out.

Ions resulting from carbon chain cleavage. Carbon chain cleavage with charge retention on the alcoholic portion of the molecule gives rise to the same series of ions with $m / e=103+\mathrm{n} \times 102$ as for alditols ${ }^{3}$ with $\mathrm{M}-117$ as the highest possible mass. These ions are formed with the deoxyaldonic acids as well where the corresponding structures are inherent in the molecules. Of the individual ions, $\mathrm{m} / \mathrm{e} 103$ is not very indicative of structure since it is often formed by rearrangement ${ }^{3}$ and easily decomposes to $m / e$ 73. The trimethylsilyl ion can probably be formed by a number of other fragmentations and gives rise to the base peak at the electron energy $70 \mathrm{eV}$ for all investigated aldonic acids and for most deoxyaldonic acids. Peaks at $m / e 205$ were found to be indicative of a vicinal diol end group. Metastable peaks indicate decomposition of the m/e 205 ion to an ion of mass 147. The m/e 147 ion in Fig 2 may be formed through a large number of alternative cyclic intermediates or transition states for most of the investigated aldonic and deoxyaldonic acids. Like the $m / e 73$ ion it gives rise to intense peaks for all the investigated acids. The structural significance of the peaks at $m / e 73$ and 147 is low, however, and the corresponding ions will not be further discussed. The $m / e 307$ ion decomposes by loss of formaldehyde to $m / e 277$ and by rearrangement loss of trimethylsilanol to the commonly encountered $m / e 217$ ion. ${ }^{3}$ Peaks from the higher analogs at $\mathrm{m} / \mathrm{e} 409,511$ and 613 are small or negligible both for alditols and aldonic acids but, as expected, rather intense peaks are obtained at $m / e 319$ (409-90) for the hexonic and heptonic acids and at $m / e 421$ (511-90) for the heptonic acid.

Ions comprising the ester portion of the molecule after carbon chain $\alpha$-cleavage exhibit the mass numbers $219+n \times 102$ with $\mathrm{M}-103$ as the highest. The formation of the $\mathrm{m} / \mathrm{e} 219$ ion is negligible because of the well-known unfavourable position of a 
positive charge adjacent to a polar carbonyl group. The low intensities of the $m / e 321$ peaks can also be attributed, at least in part, to the electron-withdrawing effect of the ester group. The most intense peaks are obtained at $m / e$ 423. The M-103 peaks are of low intensity. Metastable peaks provide evidence for the decomposition of the $\mathrm{m} / \mathrm{e} 423$ ion to the abundant $m / e 333$ ion for the aldonic acids by loss of trimethylsilanol. The analogous decomposition of $\mathrm{m} / \mathrm{e} 525$ to $\mathrm{m} / \mathrm{e} 435$ for the heptonic acid is also supported by metastable evidence. These fragmentation sequences are similar to those ${ }^{3}$ observed for the ions from the alcoholic portion of the molecule. Less significant peaks are also present at mass numbers corresponding to the loss of two molecules of trimethylsilanol from both types of $\alpha$-cleavage ions with 5 and 6 chain carbon atoms.

Ions related in mass to the molecular ion. The unfavourable effect of the ester group on the formation of some of the $\alpha$-cleavage ions may account for a less extensive fragmentation of the acids than of the alditols. The difference in stability is reflected in the higher intensities of the peaks at high mass numbers for the acids. Thus the peak at M-15 was found to be larger than $0.1 \%$ of the base peak for all investigated aldonic and deoxyaldonic acids at $70 \mathrm{eV}$, whereas it was not observed at all for pentitols and hexitols. ${ }^{3}$ Since the molecular weight can be determined from $M$ (even mass number) or M-15 (odd mass number) for all acids in $70 \mathrm{eV}$ spectra this standard electron energy was used throughout. To avoid an incorrect interpretation it should be noted that the M-15 peaks for aldonic acids fall on the $103+n \times 102$ mass number series.

Since a Me group can be lost from alternative trimethylsilyl groups, a variety of different structures for the M-15 ions are possible both for the aldonic and the deoxyaldonic acids. Only a few acids exhibit peaks at M-43 which can be explained by fragmentations similar to those shown in Fig 2 resulting in the loss of carbon monoxide. Peaks at M-15-90 from the loss of trimethylsilanol are more frequent and are found for all of the higher members. Both these decompositions of the $\mathbf{M}-15$ ions are supported by metastable peaks for several acids. A few metastable peaks also indicate step-wise loss from M-15 ions of both trimethylsilanol and carbon monoxide and peaks at M-15-118 are common. This peak (at $m / e ~ 189$ ) is unusually intense in the spectrum of glyceric acid.

In addition to the peaks related to the M-15 ions, most spectra exhibit small peaks at M-89 from loss of a trimethylsiloxyl group and at M-90 from loss of trimethylsilanol. Low intensity peaks are also encountered at $M-89-n \times 90$ as well as at M-15$\mathrm{n} \times 90$. The maximum number of trimethylsilanol molecules lost in these series corresponds to half (but not more) of the number of hydroxyl groups for almost all of the aldonic and unbranched deoxyaldonic acids. Only the most significant peaks resulting from the fragmentations discussed are indicated in the figures by their mass numbers.

The m/e 292 rearrangement ion. Glyceric acid and all aldonic acids of higher molecular weight give rise to an intense peak at $m / e 292$. The spectra of the deoxyaldonic acids discussed below disclose that formation of an abundant $m / e 292$ ion requires hydroxyl groups at C-2 and C-3 in the structure of the acid. The spectra investigated also permit the conclusion that the ion comprises the moiety on the ester side of the C-2 to C-3 bond together with a trimethylsilyl group which originates almost exclusively from the derivatized C-3 hydroxyl. The carbonyl oxygen should be the only favourable acceptor site for the rearranged TMS group. As illustrated in Fig 3 the decomposition corresponds to a McLafferty-type rearrangement with aldehyde 
loss. The extremely good resonance stabilization of the rearranged radical ion probably provides the main driving force for the reaction. The relative intensity of the $\mathrm{m} / \mathrm{e}$ 292 peak increases at lower electron energies and it was found to be the base peak at $20 \mathrm{eV}$ for many of the acids investigated. The spectra of the C-2 branched acids which are discussed below exhibit peaks at mass numbers corresponding to the mass shift caused by the side chain. The rearrangement reaction is especially noteworthy with regard to the scarcity of reported McLafferty rearrangements of groups other than hydrogen. The TMS group is known to have a high migratory aptitude in electron impact fragmentations, and long-range TMS migrations to carbonyl oxygen have been reported previously for fatty acid derivatives. ${ }^{4,16}$

Peaks at mass numbers corresponding to the conventional McLafferty rearrangement were observed for the tetronic and 4-deoxytetronic acids at $\mathrm{m} / \mathrm{e} 220$, but were negligible for all other investigated acids.

Spectra of diastereomers. As expected the differences between the spectra of diastereomeric derivatives were found to be restricted to differences in the relative intensity of various peaks for the aldonic as well as for the deoxyaldonic acids. The differences are of the same order of magnitude as for the alditol derivatives ${ }^{3}$ and increase with an increasing number of asymmetric $\mathrm{C}$ atoms.

Careful comparisons of relative intensities permit identification of pentonic and hexonic acids when reference spectra of all the diastereomers are available. The differences are not as great as for the diastereomeric aldonolactone derivatives ${ }^{1}$, however.

The discussion shows that all important features of the spectra of the open-chain aldonic acid derivatives are explained by rather uncomplicated fragmentations and a number of characteristic features permit an easy and definite identification of an unknown compound as an aldonic acid.

\section{2-Deoxyaldonic acids}

In addition to the hydracrylic acid discussed above, the following 2-monodeoxyaldonic acids were studied: 2-deoxytetronic, 2-deoxy-erythro-pentonic, 2-deoxy-arabinohexonic and 2-deoxy-lyxo-hexonic acids. The spectra of a series of these acids are given in Fig 4.

Rather intense peaks indicative of the molecular weight were found not only at $\mathrm{M}$ 15 , but also at $\mathbf{M}-90$ and $\mathbf{M}-15-90$ for all the acids except hydracrylic acid. The presence of a "deoxy group" in the molecule causes a characteristic shift in the position of these peaks compared to the aldonic acids.

The formation of chain cleavage ions for the deoxyaldonic acids is strongly influenced by the positions of the "deoxy groups". The ions exhibiting resonance stabilization of the positive charge at carbon from an adjacent ether oxygen atom ${ }^{3}$ are formed in high abundances whereas the formation of other chain cleavage ions is negligible. Thus the peaks which would result from cleavage of the $\mathrm{C}-1$ to $\mathrm{C}-2$ bond $(\mathrm{M}-117-\mathrm{n} \times 90)$ are very small or absent for the 2-deoxyaldonic acids. Most often the deoxyaldonic acids also exhibit low intensities for the oxygen stabilized ions from cleavages adjacent to a "deoxy group", probably because of a lowered stabilization of the neutral radical fragment. The low intensities of the peaks at $m / e 205$ for the 2deoxytetronic acid, at $m / e 307$ for the 2-deoxypentonic acid and at $m / e 319$ (409-90) for the 2-deoxyhexonic acids provide examples. 


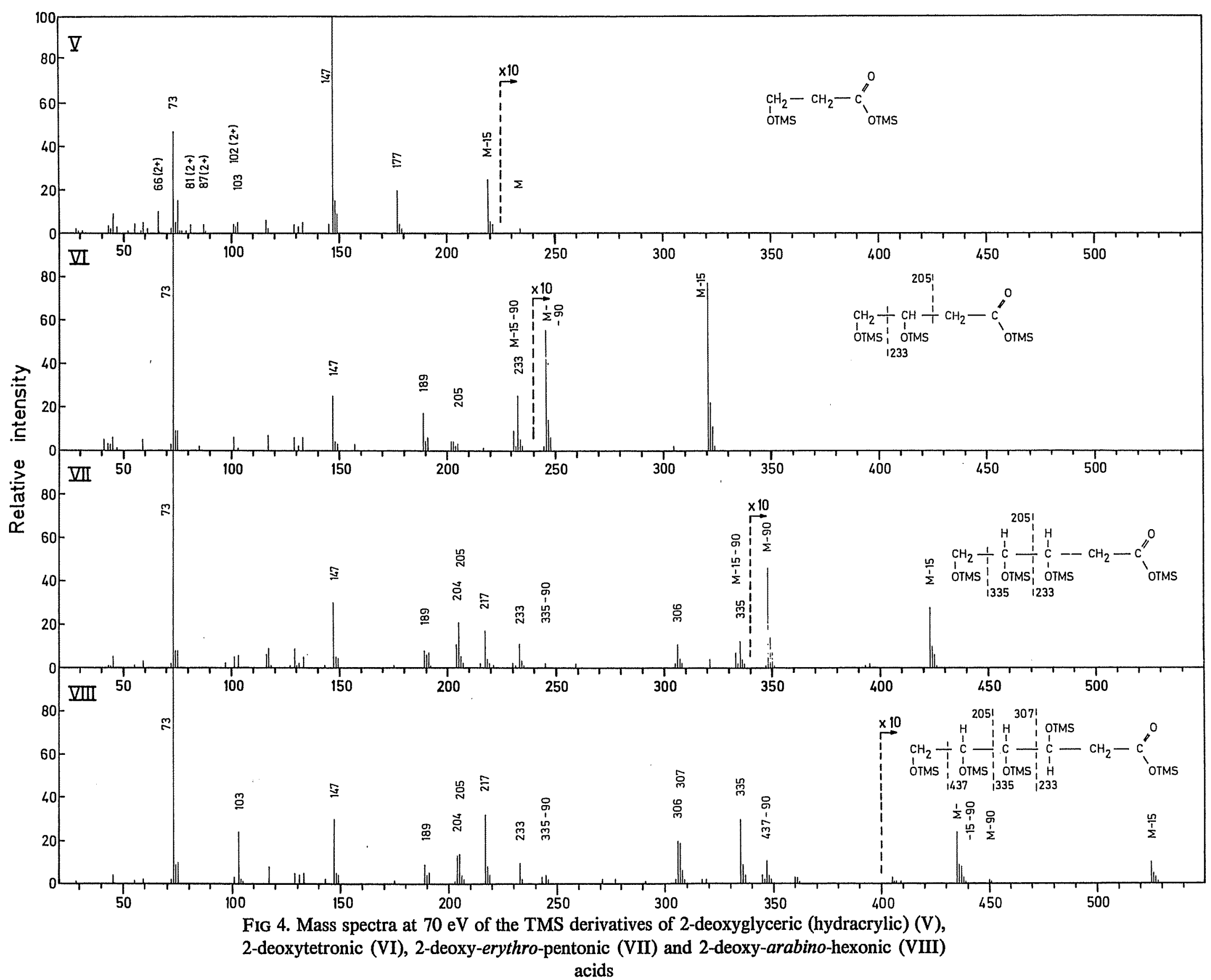




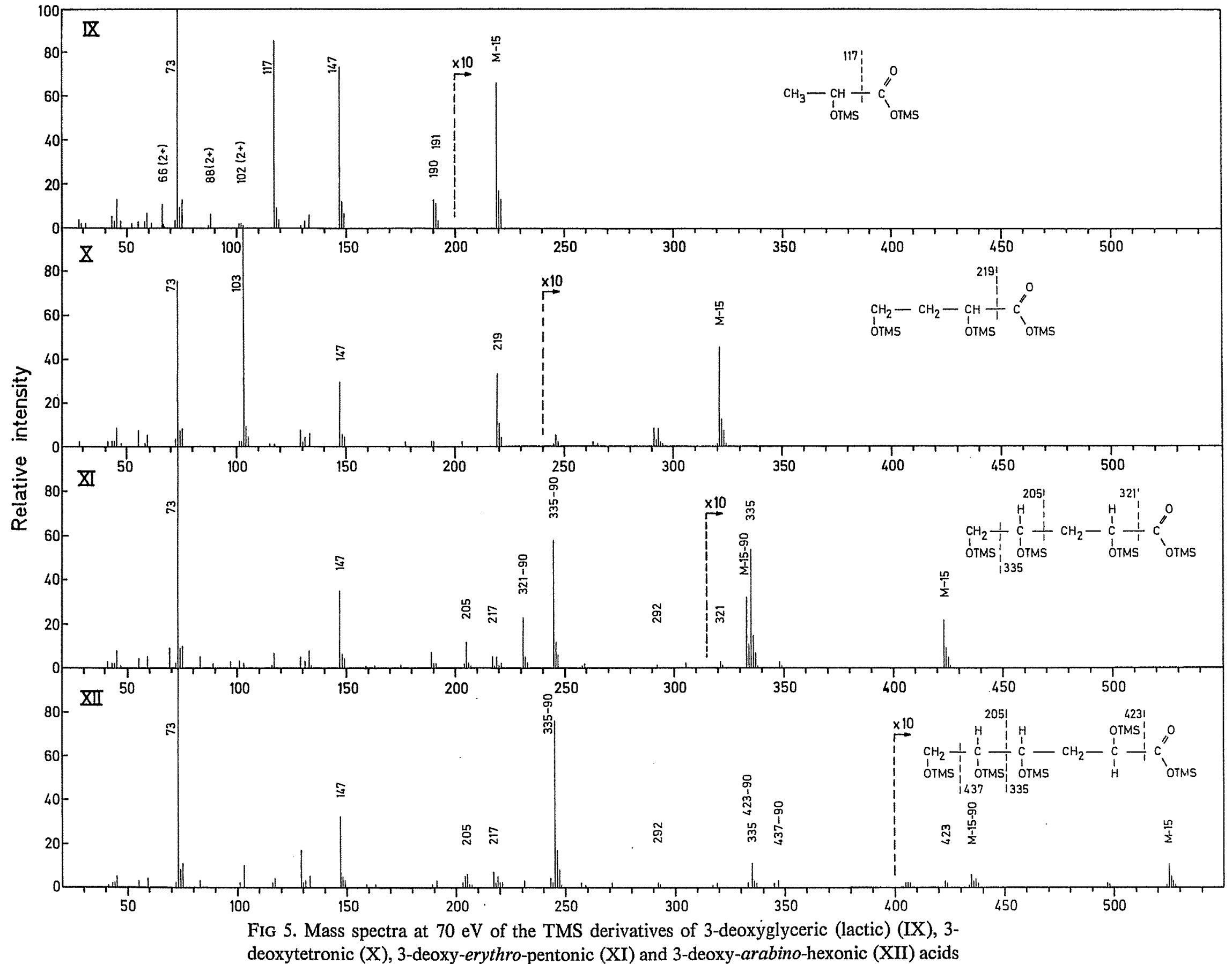


An unusually abundant ion $(m / e 335)$ is obtained from cleavage of the $\mathrm{C}-4$ to $\mathrm{C}-5$ bond. In contrast the ion of mass 245 (335-90), resulting from loss of trimethylsilanol, is formed in very low abundance. The 2-deoxyaldonic acids also give rise to an ion of mass 233 from cleavage of the $\mathrm{C}-3$ to $\mathrm{C}-4$ bond. Analogous ions from cleavage of this bond in the other unbranched acids investigated were much less abundant. A metastable peak $\left(m^{*}=153-154\right.$; calc $\left.153 \cdot 3\right)$ indicates the decomposition $m / e ~ 233 \rightarrow m / e$ 189 by loss of carbon dioxide for the 2-deoxytetronic and 2-deoxypentonic acids. This fragmentation is probably best pictured as involving migration of the ester TMS group to the oxygen atom through a six-membered transition state. The decomposition $m / e$ $307 \rightarrow m / e 277$ can be viewed in a similar way. No evidence for fragmentations by loss of ketene similar to that for hydracrylic acid was found for the other acids.

A rearrangement according to the scheme in Fig 3 would produce an ion of mass 204 for the 2-deoxyaldonic acids. Such an ion would be less effectively stabilized by resonance than the $m / e 292$ ion, but peaks of moderate intensity are actually observed at $m / e 204$ for the 2-deoxyaldonic acids. The formation of this ion of mass 204 by long-range migration of a TMS group to an ester carbonyl oxygen has been demonstrated previously. ${ }^{16}$ An isomeric $m / e ~ 204$ ion comprising two adjacent carbon atoms with derivatized hydroxyl groups can be formed by cleavage of two carbon chain bonds and gives rise to small peaks in the spectra of many of the aldonic and deoxyaldonic acias. The 2-deoxypentonic and 2-deoxyhexonic acids exhibit another intense peak from an odd-electron ion at $m / e$ 306. This ion is probably formed by a rearrangement similar to that in Fig 3, but involving a seven-membered ring since the normally preferred six-membered ring gives a structurally less favourable ion for these acids.

The most characteristic features of the spectra of the 2-deoxyaldonic acids compared with other open-chain polyhydroxy acids are the intense peaks at $m / e 233$ and $m / e 306$, and the low ratio between the intensities of the $m / e 245$ (335-90) peak and the $m / e 335$ peak. The very low intensities of the M-117- $\mathrm{n} \times 90$ peaks and the complete absence of peaks at $m / e 292$ are also characteristic.

\section{3-Deoxyaldonic acids}

The 3-deoxyaldonic acids or metasaccharinic acids are one of the main types of saccharinic acids formed on alkaline treatment of carbohydrates. Mass spectra were recorded for the TMS derivatives of 3-deoxytetronic acid, the two diastereomeric 3deoxypentonic acids and the four diastereomeric 3-deoxyhexonic acids. Spectra of acids with a different number of $\mathrm{C}$ atoms are reproduced in Fig 5 below the spectrum of lactic acid.

The influence of the methylene group upon the formation of ions from carbon chain cleavage results in a different pattern for the formation of these ions for the 3deoxyaldonic acids compared to the 2-deoxyaldonic acids. Thus abundant ions from cleavage of the $\mathrm{C}-1$ and $\mathrm{C}-2$ bond are formed, especially for the low molecular weight acids. These ions give rise to the intense peaks at $\mathrm{m} / \mathrm{e} 117$ for lactic acid, at $\mathrm{m} / \mathrm{e} 219$ for 3-deoxytetronic acid and at $m / e 231$ (321-90) for the 3-deoxypentonic acids.

The most abundant ions from the ester portion of the molecules are obtained from cleavage of the C-4 to C-5 bond for the 3-deoxyaldonic acids as well as for most of the other types of acids studied. Large metastable peaks prove the expected formation of the $\mathrm{m} / \mathrm{e} 245$ ions from the $m / e 335$ ions by loss of trimethylsilanol. Further decom- 
position of the $m / e 245$ ions to $m / e 217$ ions by loss of carbon monoxide is also indicated by metastable peaks $\left(m^{*}=192-193 \cdot 5\right.$; calc 192.2). Analogous rearrangements in even-electron ions from carbon chain cleavage are probably operative to a lesser extent for other types of acids as well, e.g. in the formation of $\mathrm{m} / \mathrm{e} 217$ ions for 2-deoxyaldonic acids and of $m / e 305$ (423-90-28) ions for aldonic acids. Similar migrations of methoxy groups to carbonium ion centres accompanied by loss of $\mathrm{CO}$ have been reported for dimethyl esters of aliphatic dicarboxylic acids. ${ }^{14}$

Most notable about the remaining peaks is the high intensity of the $\mathrm{m} / \mathrm{e} 103$ peak for the 3-deoxytetronic acid. No abundant odd-electron ions are obtained. The rearrangement ion of mass 292 (Fig 3 ) is formed in very low abundance which indicates a high specificity for the rearrangement with preference for the six-membered transition state.

The main characteristics of the spectra of the 3-deoxyaldonic acids are the peaks at M-117- $\mathrm{n} \times 90$ corresponding to cleavage of the $\mathrm{C}-1$ to $\mathrm{C}-2$ bond, the high intensities of the $m / e 335$ and especially the $m / e 245$ peaks and the low intensities of all peaks at even mass numbers.

\section{$\omega$-Deoxyaldonic acids}

The $\omega$-deoxyaldonic acids are the oxidized counterparts of the $\omega$-deoxyaldoses. These, as well as the 2-deoxyaldoses, are important deoxysugars occurring in nature. The following acids were studied: 4-deoxyerythronic, 4-deoxythreonic, 6-deoxymannonic and 6-deoxygalactonic acids.

Formation of chain cleavage ions from both ends of the molecule with identical integrate mass numbers is possible for the $\omega$-deoxyaldonic acids. A comparison with the fragmentation of the other types of acids studied permits the conclusion that the $m / e 117$ and $m / e 219$ ions are formed almost exclusively from the alcoholic portion of the molecules as indicated in the structure formula for 6-deoxymannonic acid.

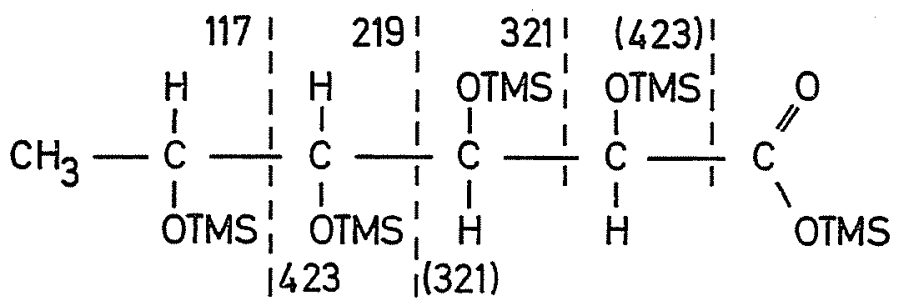

In contrast to those of lower mass the $m / e 423$ ions and the abundant $m / e 333$ (42390 ) ions should originate mainly from the ester portion of the molecule. The $m / e 117$ ion gives rise to the second most intense peak (the base peak is at $m / e$ 73) for the acids studied including lactic acid (Fig 5). With the exception of the $m / e 219$ and $m / e 333$ ions for the 6-deoxyhexonic acids, the other ions from carbon chain cleavage are formed in rather low abundance.

The $\omega$-deoxyaldonic acids fulfil the requirements (Fig 3 ) for the formation of an abundant $m / e 292$ rearrangement ion and the peak at $m / e 292$ is the most intense in the high mass region of the four spectra. The observed peak of highest mass number at M-15 gives the molecular weight. No additional significant ions were observed.

All the spectra discussed thus far demonstrate that the position of a "deoxy group" influences the formation of ions from carbon chain cleavage in a predictable way. 
Peaks from these ions should permit an easy determination of the position of the "deoxy group" for all unbranched deoxyaldonic acids. An abundant peak at $m / e 292$ should be characteristic for all unbranched acids with hydroxyl groups both at C-2 and $\mathrm{C}-3$. The $\omega$-deoxyaldonic acids are most easily recognized by the intense peak at m/e 117 .

\section{Dideoxyaldonic acids}

Two dideoxyaldonic acids were studied, 2,6-dideoxy-ribo-hexonic and 3,4dideoxypentonic acids. In some respects these acids bridge the gap to the previously investigated TMS derivatives of hydroxy acid esters. ${ }^{4-7,} 16$

The presence of a distinct peak at M-15 makes possible the determination of both the number of hydroxyl groups and the number of "deoxy groups" for all aldonic and deoxyaldonic acids. If $\mathrm{A}$ is the number of hydroxyl groups and $\mathrm{B}$ is the number of "deoxy groups", the mass number for M-15 equals $103+\mathrm{A} \times 102+\mathrm{B} \times 14$, permitting a determination of $\mathrm{A}$ and $\mathrm{B}$. Thus the peak of highest mass number at $m / e 437$ for the 2,6-dideoxyhexonic acid is compatible only with $A=3$ and $B=2$. The 3,4-dideoxypentonic acid exhibits a distinct peak at $m / e 350$ from the molecular ion in addition to the prominent peak at M-15.

The spectrum of the 2,6-dideoxyhexonic acid can be regarded as being composed of features characteristic of the 2-deoxyaldonic acids and of the $\omega$-deoxyaldonic acids.

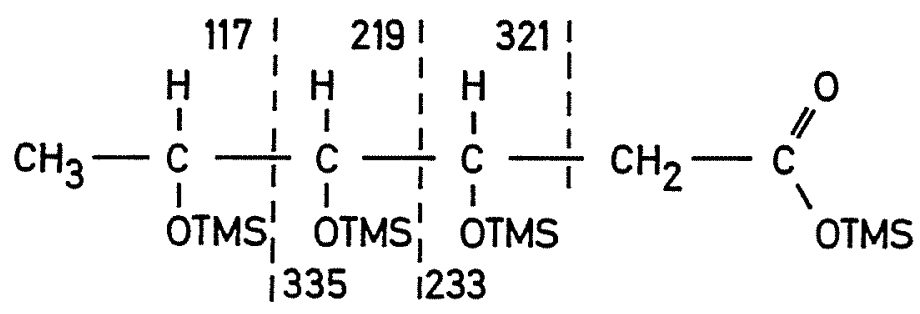

The chain cleavage ions from the alcoholic portion of the molecule give rise to peaks similar to those of the 6-deoxyhexonic acids at $m / e$ 117, 219 and 231 (321-90). The peak at $m / e 117$ is the second most intense in the spectrum after the $m / e 73$ peak. The ions of mass 233, 335 and 245 (335-90) from the ester portion of the molecule are formed in about the same abundance as those of the analogous ions in the 2deoxyhexonic acids. Prominent peaks at $\mathrm{m} / \mathrm{e}$ 204, 217 and 306 can also be attributed to the same ions as for the 2-deoxyaldonic acids. No other significant peaks are encountered for the 2,6-dideoxyhexonic acid.

As could be anticipated from the discussions of chain cleavage ions, only one ion of importance results from a simple cleavage of the carbon chain of 3,4-dideoxypentonic acid.

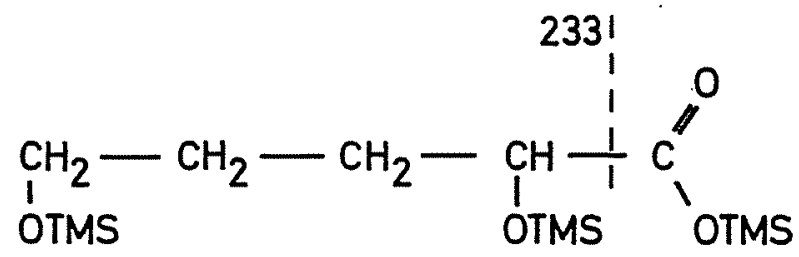

The peak at $m / e 233$ is the most intense in the upper mass range of the spectrum and furthermore a peak at $m / e 143(233-90)$ constitutes the base peak. The peaks at $m / e 73$ 


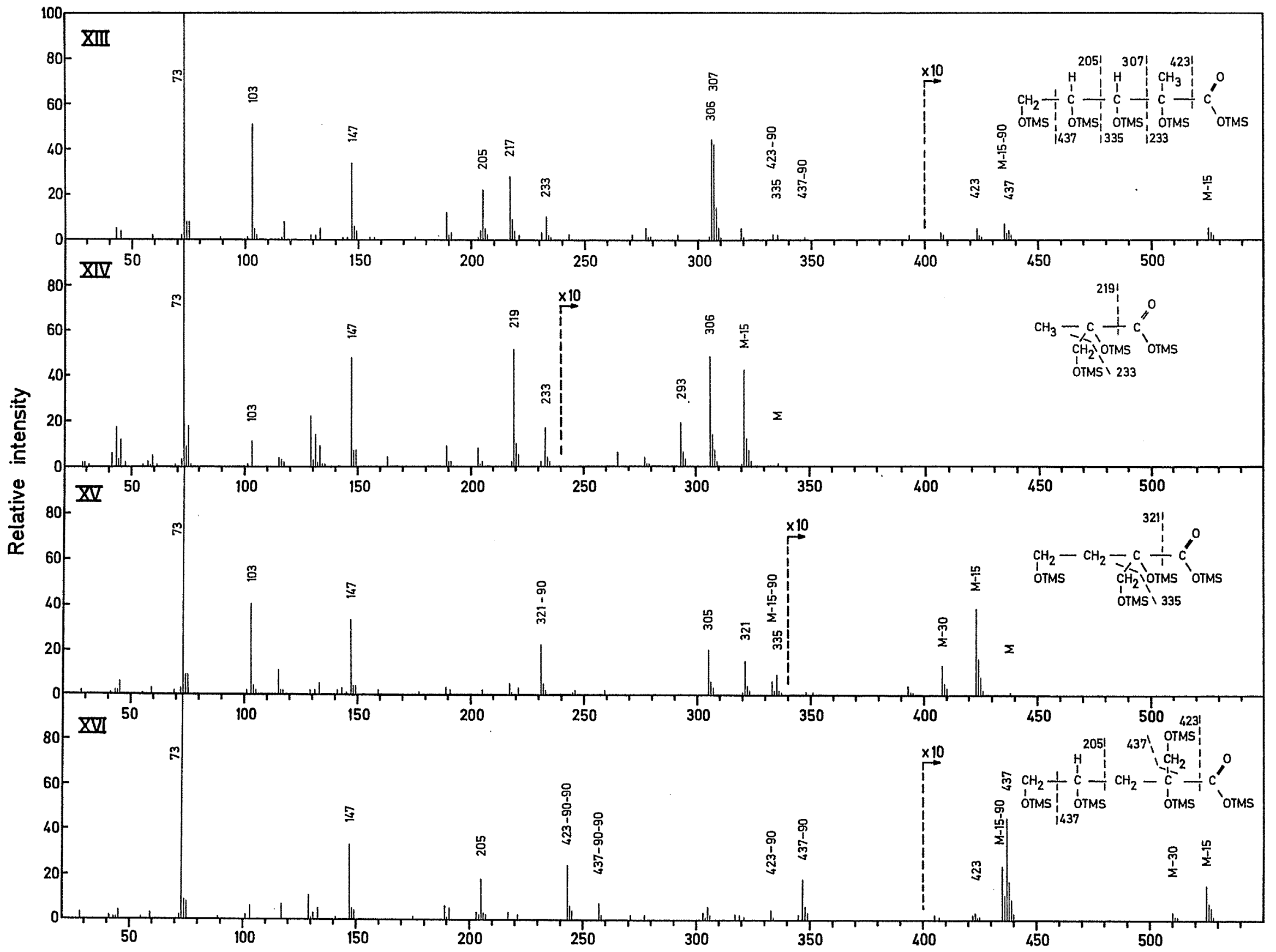

FIG 6. Mass spectra at $70 \mathrm{eV}$ of the TMS derivatives of 2-C-methylribonic (XIII), 2-Cmethylglyceric (XIV), 3-deoxy-2-C-(hydroxymethyl)tetronic (XV) and 3-deoxy-2-Chydroxymethyl-erythro-pentonic (XVI) acids 
and $m / e 147$ are the second and third most intense peaks. The only remaining prominent peak $(m / e ~ 71)$ might be due to a tetrahydrofuryl ion formed by cyclization.

The dideoxyaldonic acids studied illustrate further the excellent opportunities offered by mass spectrometry for the determination of the position of hydroxyl groups in hydroxy acids.

\section{Branched-chain saccharinic acids}

The two best known types of branched saccharinic acids are the 3-deoxy-2-C(hydroxymethyl)aldonic acids, termed isosaccharinic acids, and the 2-C-methylaldonic acids. Mass spectra were recorded for 2-C-methylribonic (" $\alpha$ "-glucosaccharinic) acid, 2-C-methylglyceric acid, 3-deoxy-2-C-(hydroxymethyl)tetronic acid, 3-deoxy-2-Chydroxymethyl-erythro-pentonic (" $\rho$ "-isosaccharinic) and 3-deoxy-2-C hydroxymethyl-threo-pentonic (" $\beta$ "-isosaccharinic) acids. The configurations of the diastereomeric six-carbon isosaccharinic acids have been determined recently. ${ }^{17}$ Representative spectra of four branched saccharinic acids are reproduced in Fig 6.

A comparison of the spectra of 2-C-methylribonic and 2-C-methylglyceric acids in Fig 6 with those of arabinonic and glyceric acids in Fig 1 clearly demonstrates analogous fragmentations for 2-C-methylaldonic acids and aldonic acids. The shift of $14 \mathrm{mu}$ towards higher mass numbers for the ions comprising the $\mathrm{C}$-methyl group of the 2-C-methylaldonic acids explains most of the differences in the spectra. The chain cleavage ions with charge retention at $\mathrm{C}-2$ exhibit charge stabilization from the methyl group for the 2-C-methylaldonic acids. This is reflected in higher intensities for the peaks at $\mathrm{m} / \mathrm{e} 233$ and 219 for 2-C-methylglyceric acid and at $\mathrm{m} / \mathrm{e} 233$ and 423 for 2-Cmethylribonic acid compared with the analogous peaks for the aldonic acids. The presence of intense peaks at $m / e 306$ for the 2-C-methylaldonic acids instead of the peaks at $m / e 292$ for aldonic acids is consistent with the scheme in Fig 3 for the formation of the $m / e$ ion. 292 ion.

Since 2-C-methylglyceric acid can be considered 3-deoxy-2-C-(hydroxymethyl)glyceric acid the last three acids in Fig 6 form a series of 3-deoxy-2C-(hydroxymethyl)aldonic acids from which the most important fragmentation characteristics can be deduced. A trimethylsiloxymethyl group (103 mu) can be lost from two alternative positions for the acids with six $\mathrm{C}$ atoms. The ion with charge localization at C-2 should be the less favourable of the two isomeric $m / e 437$ ions. Further loss of trimethylsilanol provides an obvious explanation of the peaks at $\mathrm{m} / \mathrm{e} 347$ (43790 ) and 257 (437-2 $\times 90)$. For the acid with five $C$ atoms, an M-103 ion with charge stabilization from an ether oxygen adjacent to the site of the charge can be formed only by loss of the side group. Cleavage of the $\mathrm{C}-1$ to $\mathrm{C}-2$ bond gives rise to abundant ions of mass 321 and 231 (321-90) for the five-carbon acid and of mass 333 (423-90) and $243(423-2 \times 90)$ for the 6-carbon acids. The decompositions $m / e 321 \rightarrow m / e$ 231 and $m / e 333 \rightarrow m / e 243$ are corroberated by strong metastable peaks. Formation of $m / e 321$ and $m / e 231$ ions from the other end of the molecule by cleavage of the C-2 to $\mathrm{C}-3$ bond is probably negligible since the corresponding peaks are very small for the 6-carbon acids.

In contrast to most of the other acids the four isosaccharinic acids studied exhibit distinct peaks (about $0.1 \%$ of the base peak) from the molecular ions. This, as well as the high intensities of the M-15 peaks, can be ascribed to the fewer favoured fragmentation paths for the isosaccharinic acids. A metastable peak indicates that the $\mathrm{m} / \mathrm{e}$ 
293 ions for the four-carbon acid are formed from M-15 ions by the loss of CO (cf. Fig 2). The ions of mass 305 (M-15-90-28) for the 5-carbon acid might also be expected to arise from $\mathrm{M}-15$ ions. A metastable peak $\left(m^{*}=277.5-279\right.$; calc 277.7$)$ demonstrates, however, that these ions are formed at least in part from the $m / e$ 335 ion, probably by analogy with the decomposition $m / e 307 \rightarrow m / e ~ 277$ for aldonic acids.

A characteristic feature of the spectra of isosaccharinic acids is the peaks at M-30. If the acids are regarded as C-2-branched glyceric acids, it is evident that loss of formaldehyde from the molecular ions is to be expected by analogy with the rearrangement depicted in Fig 3. The M-30 ions exhibit rather low intensities, however.

The spectra studied demonstrate that 2-C-methylaldonic acids and 3-deoxy-2-C(hydroxymethyl)aldonic acids can be recognized from characteristic features of their mass spectra. Good opportunities to distinguish branched deoxyaldonic acids from all unbranched are also indicated. The most useful ions for conclusions about the structure of branched deoxyaldonic acids seem to be the ions from carbon chain cleavage and the ions from the specific rearrangement of a TMS group.

\section{EXPERIMENTAL}

Most of the acids have been prepared in connection with previously published work from this department. Some acids were obtained from commercial sources. 3-Deoxy-2-C-(hydroxymethyl)tetronic acid was kindly supplied by Dr. O. Theander, Svenska Träforskningsinstitutet, Stockholm.

TMS derivatives were prepared from suspensions in anhydrous pyridine of $1-10 \mathrm{mg}$ of the $\mathrm{Na}$, $\mathrm{Ca}$ or Ba salts of the acids. Excess bis(trimethylsilyl)acetamide (BSA) and half the volume trimethylsilylchlorosilane (TMCS) were used as reagents and the mixture was shaken for a few hours to complete the reaction. After evaporation in a rotary vacuum evaporator the residue was dissolved in diethyl ether. To avoid interference from BSA peaks in gas chromatography, hexamethyldisilazane (HMDS) and TMCS were used for derivatization of the free acid form of some of the lower acids.

The mass spectrometric measurements were performed on a LKB 9000 gas chromatograph-mass spectrometer. 1-10 $\mu \mathrm{g}$ of the TMS derivatives were introduced through a QF-1 column. The column temperatures were in the interval $80-180^{\circ}$ and the spectra were corrected for the low column bleeding. The temp of the molecule separator was $205-220^{\circ}$ and that of the ion source $270^{\circ}$.

Acknowledgement-The financial support of the Swedish Council for Applied Research is gratefully acknowledged.

\section{REFERENCES}

${ }^{1}$ G. Petersson, O. Samuelson, K. Anjou and E. von Sydow, Acta Chem. Scand. 21, 1251 (1967)

${ }^{2}$ G. Petersson, H. Riedl and O. Samuelson, Svensk Papperstidn. 70, 371 (1967)

${ }^{3}$ G. Petersson, Tetrahedron, 25, 4437 (1969)

${ }^{4}$ P. Capella and C. M. Zorzut, Analyt. Chem. 40, 1458 (1968)

${ }_{5}^{5}$ P. Capella, C. Galli and R. Fumagalli, Lipids 3, 431 (1968)

${ }^{6}$ G. Eglinton and D. H. Hunneman, Phytochem. 7, 313 (1968)

7 G. Eglinton, D. H. Hunneman and K. Douraghi-Zadeh, Tetrahedron 24, 5929 (1968)

8 R. L. Brunelle, R. L. Schoeneman and G. E. Martin, J. Assoc. Offic. Agr. Chemists 50, 329 (1967)

9 O. Raunhardt, H. W. H. Schmidt and H. Neukom, Helv. Chim. Acta 50, 1267 (1967)

10 J. Diekman, J. B. Thomson and C. Djerassi, J. Org. Chem. 32, 3904 (1967)

11 A. G. Sharkey, Jr., R. A. Friedel and S. H. Langer, Analyt. Chem. 29, 770 (1957)

12 J. Diekman, J. B. Thomson and C. Djerassi, J. Org. Chem. 33, 2271 (1968)

13 J. O: Madsen, S.-O. Lawesson, J. H. Bowie and R. G. Cooks, Chem. Commun. 698 (1968)

14 I. Howe and D. H. Williams, J. Chem. Soc. (C) 202 (1968)

is S.-O. Lawesson, L. Dalgaard, J. O. Madsen, J. H. Bowie and D. B. Cobb, Chem. Commun. 218 (1969)

16 G. H. Draffan, R. N. Stillwell and J. A. McCloskey, Org. Mass Spectrom. 1, 669 (1968)

${ }_{17}$ M. von Glehn, P. Kierkegaard, R. Norrestam, O. Rönnquist and P.-E. Werner, Chem. Commun. 291 (1967) 